\title{
Periodic Solutions with Prescribed Minimal Period for $2 n$ th-Order Nonlinear Discrete Systems
}

\author{
Haiping Shi $\mathbb{D}^{1}{ }^{1}$ Peifang Luo, ${ }^{1}$ and Zan Huang ${ }^{2}$ \\ ${ }^{1}$ Department of Basic Courses, Guangzhou Maritime University, Guangzhou 510725, China \\ ${ }^{2}$ School of Naval Architecture and Ocean Engineering, Guangzhou Maritime University, Guangzhou 510725, China \\ Correspondence should be addressed to Haiping Shi; shp7971@163.com
}

Received 25 June 2021; Accepted 13 August 2021; Published 6 September 2021

Academic Editor: Douglas R. Anderson

Copyright (c) 2021 Haiping Shi et al. This is an open access article distributed under the Creative Commons Attribution License, which permits unrestricted use, distribution, and reproduction in any medium, provided the original work is properly cited.

In this paper, by using the critical point theory, some new results of the existence of at least two nontrivial periodic solutions with prescribed minimal period to a class of $2 n$ th-order nonlinear discrete system are obtained. The main approach used in our paper is variational technique and the linking theorem. The problem is to solve the existence of periodic solutions with prescribed minimal period of $2 n$ th-order discrete systems.

\section{Introduction}

In the following and in the sequel, we denote by $\mathbb{N}, \mathbb{Z}$, and $\mathbb{R}$ the sets of all natural numbers, integers, and real numbers, respectively. The symbol $*$ is defined by the transpose of a vector. Let $[\cdot]$ be the greatest integer function. For any integers $a$ and $b$ with $a \leq b$, we let $\mathbb{Z}(a, b)$ denote the discrete interval $\{a, a+1, \ldots, b\}$ and $\mathbb{Z}(a)=\{a, a+1, \ldots$,$\} .$

Now, we concern with the following $2 n$ th-order nonlinear discrete system:

$$
\Delta^{2 n} x_{k-n}=(-1)^{n} g\left(k, x_{k}\right), \quad n \in \mathbb{Z}(3), k \in \mathbb{Z},
$$

where $\Delta$ is the forward difference operator defined by $\Delta x_{k}=x_{k+1}-x_{k}, \Delta^{0} x_{k}=x_{k}$, and $\Delta^{j} x_{k}=\Delta^{j-1}\left(\Delta x_{k}\right)$ for $j \geq 2$, $g(t, x) \in C^{1}\left(\mathbb{R}^{2}, \mathbb{R}\right)$, and $g(t+M, x)=g(t, x)$ for a given integer $M \geq 3$.

We may think of (1) as a discrete analogue of the following $2 n$ th-order differential equation:

$$
\frac{\mathrm{d}^{2 n} x(t)}{\mathrm{d} t^{2 n}}=(-1)^{n} g(t, x(t)), \quad t \in \mathbb{R} .
$$

Equations similar in structure to (2) have been studied by many authors [1-6]. The difficulty of this paper comparing with system (2) is that there are few known techniques for studying the existence of periodic solutions with minimal period of (1).

Denote

$$
\omega=\frac{2 \pi}{M}
$$

Throughout this paper, we suppose that there is a function $G(t, x) \in C^{2}\left(\mathbb{R}^{2}, \mathbb{R}\right)$ with $G(t+M, x)=G(t, x)$, $G(-t,-x)=G(t, x), G(t, x) \geq 0$, and

$$
\frac{\partial G(t, x)}{\partial x}=g(t, x), \quad \forall t \in \mathbb{R} \text {. }
$$

Bin [7] in 2013 considered the second-order discrete Hamiltonian systems:

$$
\Delta x_{k-1}+W^{\prime}\left(k, x_{k}\right)=0, \quad x_{k+M}=x_{k}
$$

By using Morse theory, some new results concerning the existence of nontrivial periodic solution are obtained.

Using the critical point method, Liu et al. [8] studied the following forward and backward difference equation:

$$
\Delta^{n}\left(r_{k-n} \Delta^{n} x_{k-n}\right)=(-1)^{n} f\left(k, x_{k+1}, x_{k}, x_{k-1}\right), \quad n \in \mathbb{Z}(3), k \in \mathbb{Z} .
$$

Some new criteria for the existence and multiplicity of periodic and subharmonic solutions are established. 
In 2016, Leng [9] established some new criteria for the existence and multiplicity of periodic and subharmonic solutions to the $2 n$ th-order difference equation with $\phi_{c}$-Laplacian

$$
\Delta^{n}\left(r_{k-n} \phi_{c}\left(\Delta^{n} x_{k-n}\right)\right)=(-1)^{n} f\left(k, x_{k+1}, x_{k}, x_{k-1}\right), \quad k \in \mathbb{Z},
$$

using the linking theorem in combination with variational technique.

By establishing a new proper variational framework and using the critical point theory, $\mathrm{He}$ [10] established some new existence criteria to guarantee that the $2 n$ th-order nonlinear difference equation containing both advance and retardation with $p$-Laplacian

$$
\Delta^{n}\left(r_{k-n} \varphi_{p}\left(\Delta^{n} x_{k-1}\right)\right)+q_{n} \varphi_{p}\left(x_{k}\right)=f\left(k, x_{k+n}, \ldots, x_{k}, \ldots, x_{k-n}\right), \quad n \in \mathbb{Z}(3), k \in \mathbb{Z},
$$

has infinitely many homoclinic orbits.

Lin and Zhou [11] concerned with the existence and multiplicity of periodic and subharmonic solutions of the following $2 n$ th-order difference equation

$$
\Delta^{n}\left(r_{k-n} \phi\left(\Delta^{n} x_{k-1}\right)\right)=(-1)^{n} f\left(k, x_{k+n}, \ldots, x_{k}, \ldots, x_{k-n}\right), \quad k \in \mathbb{Z} .
$$

By using the critical point theory, some new sufficient conditions are obtained and some previous results are generalized.

Xia [12] in 2018 considered the existence of periodic solutions for a higher order nonlinear difference equation

$$
\sum_{i=0}^{n} r_{i}\left(X_{k-i}+X_{k+i}\right)+f\left(k, X_{k+\Gamma}, \ldots, X_{k}, \ldots, X_{k-\Gamma}\right)=0, \quad n \in \mathbb{N}, k \in \mathbb{Z}
$$

by making use of the critical point theory. Some existence criteria are established. Some results are generalized.

In 1978, Rabinowitz [13] proposed a conjecture that the Hamiltonian system has a nonconstant periodic solution with prescribed minimal period under some given conditions. From then on, there has been much progress [14-16] on Rabinowitz's conjecture under various conditions. Ambrosetti and Mancini [14] assumed that the dual functional is bounded from below and the Hamiltonian system has a minimum to which correspond a solution with given minimal period. Ekeland and Hofer [16] proved that if the Hamiltonian system is flat near an equilibrium and superquadratic near infinity, it has a periodic solution with minimal period. The estimate of number of periodic solutions was established in [15]. In contrast to differential equations, the research on periodic solutions with prescribed minimal period of higher order discrete systems is fresh and there are very few literature (see [7-12, 17-29]) on it. Comparing this paper with references [8-11], the advantages and differences of this paper are that the existence of periodic solutions with prescribed minimal period of (1) is obtained in this paper; however, only periodic solutions are obtained in the references [8-11]. Given integer $T \geq 2$, Long [23] considered the following $T$-cycle discrete Hamiltonian systems:

$$
J \Delta x_{k}=\nabla H\left(x, L x_{k}\right) .
$$

By making use of minimax theory and geometrical index theory, some results on the existence and multiplicity of subharmonic solutions with prescribed minimal period to the abovementioned discrete Hamiltonian systems are obtained. Yu et al. [27] in 2004 obtained some sufficient conditions on the existence of subharmonic solutions with prescribed minimal period for the second-order difference equation by using variational methods. Therefore, there is still spacious room to explore the periodic solutions with prescribed minimal period of higher order discrete systems. Motivated by the papers $[9,12]$, for a given integer $s$ with $s>1$, the aim of this paper is to obtain some new results for the existence of at least two nontrivial periodic solutions with minimal period $s M$ to a $2 n$ th-order discrete system by using critical point method.

Here, we give the existence results of at least two nontrivial periodic solutions with minimal period $s M$ as follows.

Theorem 1. Suppose that $G(t, x)$ satisfies the following assumptions:

$\left(G_{1}\right) \quad$ There are constants $\delta_{1}>0$ and $\rho \in\left(0,\left(\left(4 \sin ^{2}(\pi / s M)\right)^{n} / 2\right)\right)$ such that

$$
G(t, x) \leq \rho x^{2}, \quad \forall t \in \mathbb{Z},|x| \leq \delta_{1} .
$$

$\left(G_{2}\right)$ There are constants $\tau>0$ and $\rangle \in\left(\left(4^{n} / 2\right),+\infty\right)$ such that

$$
G(t, x) \geq>x^{2}-\tau, \quad \forall(t, x) \in \mathbb{R}^{2}
$$

$\left(G_{3}\right)$ There are three constants $\kappa>0$ and $C>D>0$ such that 


$$
\begin{aligned}
& \left(\frac{\partial^{2} G(t, x)}{\partial x^{2}} \xi, \xi\right) \leq C \xi^{2}, \quad \forall(t, x) \in \mathbb{R}^{2}, \xi \in \mathbb{R}, \\
& \left(\frac{\partial^{2} G(t, x)}{\partial x^{2}} \xi, \xi\right) \geq D \xi^{2}, \quad \forall|x| \leq \kappa, s \in \mathbb{R}, \xi \in \mathbb{R} .
\end{aligned}
$$

$\left(G_{4}\right)$ If $₫$ is a rational number, $x$ is a solution of $(1)$ with a minimal period $\omega M$, and $g(t, x)$ also has a minimal period $\omega M$, then $\omega$ must be an integer.

$\left(G_{5}\right)$ Denote by $m_{s}$ the least prime factor of $s$ :

$$
\begin{aligned}
& \left(4 \sin ^{2} \frac{\omega m_{s}}{2 s}\right)^{n}>C, \\
& \left(4 \sin ^{2} \frac{\omega}{2 s}\right)^{n}<D, \\
& \sum_{k=1}^{s M} g^{2}(k, 0)<\frac{4 \pi \kappa^{2}\left[\left(4 \sin ^{2}\left(\omega m_{s} / 2 s\right)\right)^{n}-C\right]\left[D-\left(4 \sin ^{2}(\omega / 2 s)\right)^{n}\right]}{\omega} .
\end{aligned}
$$

Then, (1) possesses at least two nontrivial periodic solutions with minimal period $s M$.

Corollary 1. Suppose that $G(t, x)$ satisfies $\left(G_{1}\right)-\left(G_{4}\right)$ and

$$
\begin{aligned}
& \left(4 \sin ^{2} \frac{\omega}{2}\right)^{n}>C \\
& \left(4 \sin ^{2} \frac{\omega}{2 s}\right)^{n}<D .
\end{aligned}
$$

If

$$
\sum_{k=1}^{s M} g^{2}(k, 0)<\frac{4 \pi \kappa^{2}\left[\left(4 \sin ^{2}(\omega / 2)\right)^{n}-C\right] D}{\omega},
$$

then there is $S>0$ such that for any prime integer $s>S$, (1) possesses at least two nontrivial periodic solutions with minimal period $s M$.

Theorem 2. Suppose that $G(t, x)$ satisfies $\left(G_{3}\right)-\left(G_{5}\right)$ and the following assumptions:

$\left(G_{6}\right) \lim _{|x| \longrightarrow+\infty}\left(G(t, x) / x^{2}\right)=0$ uniformly for $t \in \mathbb{R}$.

$\left(G_{7}\right)$ There are constants $\delta_{2}>0$ and $\theta>2$ such that

$$
0<\theta G(t, x) \leq x g(t, x), \quad \forall t \in \mathbb{Z},|x| \leq \delta_{2} .
$$

Then, (1) has at least two nontrivial periodic solutions with minimal period $s M$.

Theorem 3. Suppose that $G(t, x)$ satisfies $\left(G_{1}\right)-\left(G_{4}\right)$ and the following assumptions. If

$$
\begin{aligned}
& g(k, 0)=0, \quad \forall k \in \mathbb{Z}, \\
&\left(4 \sin ^{2} \frac{\omega m_{s}}{2 s}\right)^{n}>C, \\
&\left(4 \sin ^{2} \frac{\omega}{2 s}\right)^{n}<D,
\end{aligned}
$$

then (1) has at least two nontrivial periodic solutions with minimal period $s M$.

Corollary 2. Suppose that $G(t, x)$ satisfies $\left(G_{1}\right)-\left(G_{4}\right)$ and the following assumptions. If

$$
\begin{aligned}
& g(k, 0)=0, \quad \forall k \in \mathbb{Z}, \\
&\left(4 \sin ^{2} \frac{\omega}{2}\right)^{n}>C, \\
&\left(4 \sin ^{2} \frac{\omega}{2 s}\right)^{n}<D .
\end{aligned}
$$

then there is $S>0$ such that for any prime integer $s>S$, (1) possesses at least two nontrivial periodic solutions with minimal period $s M$.

The remainder of this paper is organized as follows. In Section 2, we build the variational functional and gather some basic notations that are necessary in the proofs of our main theorems. In Section 3, we state some useful lemmas. In Section 4, the main results will be proved.

Regarding the basis for variational methods, we refer the reader to [30]. Regarding the basic knowledge of integral inequalities and extended hypergeometric functions, the reader is referred to [31].

\section{Preliminaries}

In this section, we shall establish the variational framework associated with (1) and gather some basic notations that are necessary in the proofs of our main theorems.

Let the vector space $\mathbb{B}$ be defined by

$$
\mathbb{B}=\left\{x \mid x_{k+s M}=x_{k}\right\},
$$

and for any $x \in \mathbb{B}$, define the inner product

$$
(x, y)=\sum_{i=1}^{s M} x_{i} y_{i}
$$


and the norm

$$
\|x\|=\left(\sum_{i=1}^{s M} x_{i}^{2}\right)^{1 / 2} .
$$

For any $x \in \mathbb{B}$, let $I$ be the functional defined by

$$
I(x)=\frac{1}{2} \sum_{k=1}^{s M}\left(\Delta^{n} x_{k-1}\right)^{2}-\sum_{k=1}^{s M} G\left(k, x_{k}\right)
$$

Then, $I$ is continuously differentiable and

$$
\frac{\partial I}{\partial u_{k}}=(-1)^{n} \Delta^{2 n} x_{k-n}-g\left(k, x_{k}\right), \quad \forall k \in \mathbb{Z}(1, s M) .
$$

Thus, $x$ is a critical point of $I(x)$ on $\mathbb{B}$ if and only if

$$
\Delta^{2 n} x_{k-n}=(-1)^{n} g\left(k, x_{k}\right), \quad \forall k \in \mathbb{Z}(1, s M) .
$$

Therefore, we reduce the problem of finding $s M$-periodic solutions of (1) to that of seeking critical points of the functional $I$ on $\mathbb{B}$.

Denote

$$
L=\left(\begin{array}{cccccc}
2 & -1 & 0 & \cdots & 0 & -1 \\
-1 & 2 & -1 & \cdots & 0 & 0 \\
0 & -1 & 2 & \cdots & 0 & 0 \\
\cdots & \cdots & \cdots & \cdots & \cdots & \cdots \\
0 & 0 & 0 & \cdots & 2 & -1 \\
-1 & 0 & 0 & \cdots & -1 & 2
\end{array}\right)_{s M \times s M}
$$

It is clear that the eigenvalues of $L$ are

$$
\lambda_{i}=4 \sin ^{2} \frac{i \pi}{s M}, \quad i=0,1,2, \ldots, s M-1 .
$$

Furthermore, $L$ is positively semidefinite and all of eigenvalues of $L$ are positive except for 0 , and

$$
\begin{aligned}
& \min \left\{\lambda_{1}, \lambda_{2}, \ldots, \lambda_{s M-1}\right\}=4 \sin ^{2} \frac{\pi}{s M}, \\
& \max \left\{\lambda_{1}, \lambda_{2}, \ldots, \lambda_{s M-1}\right\} \leq 4
\end{aligned}
$$

Obviously, 0 is an eigenvalue of $L$ and $(1,1, \ldots, 1)^{*}$ is an eigenvector associated to 0 . Let $A=\left\{(c, c, \ldots, c)^{*} \in \mathbb{B} \mid c \in\right.$ $\mathbb{R}\}$, then $A$ is an invariant subspace of $\mathbb{B}$. Denote $B$ by $\mathbb{B}=A \oplus B$.

The eigenvectors of $L$ corresponding to $\lambda_{i}$ are defined by

$$
\begin{aligned}
& \alpha_{i}=\left(\cos \frac{2 i \pi}{s M}, \cos \frac{2 i \pi \cdot 2}{s M}, \ldots, \cos \frac{2 i \pi \cdot s M}{s M}\right)^{*}, \quad i=1,2, \ldots,\left[\frac{s M-1}{2}\right], \\
& \beta_{i}=\left(\sin \frac{2 i \pi}{s M}, \sin \frac{2 i \pi \cdot 2}{s M}, \ldots, \sin \frac{2 i \pi \cdot s M}{s M}\right)^{*}, \quad i=1,2, \ldots,\left[\frac{s M-1}{2}\right] . \\
& x_{i}=p+\sum_{k=1}^{[(s M-1) / 2]}\left(p_{k} \cos \frac{\omega k}{s} i+q_{k} \sin \frac{\omega k}{s} i\right),
\end{aligned}
$$

$$
\begin{aligned}
& Y=\operatorname{span}\left\{\alpha_{i}\right\}, \quad i=1,2, \ldots,\left[\frac{s M-1}{2}\right], \\
& Z=\operatorname{span}\left\{\beta_{i}\right\}, \quad i=1,2, \ldots,\left[\frac{s M-1}{2}\right] .
\end{aligned}
$$

If $s M$ is odd, then $\mathbb{B}=A \oplus Y \oplus Z$. For any $x \in \mathbb{B}$ and $i \in \mathbb{Z}$,

$$
x_{i}=p+(-1)^{i} q+\sum_{k=1}^{[(s M-1) / 2]}\left(p_{k} \cos \frac{\omega k}{s} i+q_{k} \sin \frac{\omega k}{s} i\right) \text {, }
$$

where $p, q, p_{k}$, and $q_{k}$ are constants.

If $s M$ is even, then 4 is the eigenvalue of $L$. Let $\eta$ denote the eigenvector corresponding to 4 , and $W=\operatorname{span}\{\eta\}$. We have $\mathbb{B}=A \oplus Y \oplus Z \oplus W$. For any $x \in \mathbb{B}$ and $i \in \mathbb{Z}$, where $p, p_{k}$, and $q_{k}$ are constants.

Suppose that $\mathbb{B}$ is a real Banach space and $I \in C^{1}(\mathbb{B}, \mathbb{R})$. As usual, $I$ is said to satisfy the Palais-Smale condition if every sequence $\left\{x^{(j)}\right\} \subset \mathbb{B}$, such that $\left\{I\left(x^{(j)}\right)\right\}$ is bounded and $I^{\prime}\left(x^{(j)}\right) \longrightarrow 0,(j \longrightarrow \infty)$, has a convergent subsequence. The sequence $\left\{x^{(j)}\right\}$ is called a Palais-Smale sequence.

\section{Some Lemmas}

To apply critical point theory to study the existence of periodic solutions with minimal period $s M$ of (1), some lemmas should be stated in this section which will be used in proofs of our main results. 
Below, we denote by $B_{r}(x)$ the open ball centered at $x \in \mathbb{B}$ with radius $r>0, \bar{B}_{r}(x)$ as its closure, and $\partial B_{r}(x)$ as its boundary.

Lemma 1 (linking theorem [30]). Let $\mathbb{B}$ be a real Banach space, $\mathbb{B}=\mathbb{B}_{1} \oplus \mathbb{B}_{2}$, where $\mathbb{B}_{1}$ is finite dimensional. Suppose that $I \in C^{1}(\mathbb{B}, \mathbb{R})$ satisfies the Palais-Smale condition and the following:

$\left(I_{1}\right)$ There are positive constants $c$ and $r$ such that $\left.I\right|_{\partial B_{r}(0) \cap \mathbb{B}_{2}} \geq c$

$\left(I_{2}\right)$ There is $\mu \in \partial B_{1}(0) \cap \mathbb{B}_{2}$ and a positive constant $\widehat{c} \geq r \quad$ such that $\left.I\right|_{\partial \Omega} \leq 0, \quad$ where $\Omega=\left(\bar{B}_{\widehat{c}}(0) \cap \mathbb{B}_{1}\right) \oplus\{t \mu \mid 0<t<\widehat{c}\}$

Then, I possesses a critical value $c_{0} \geq c$, where

$$
c_{0}=\inf _{d \in \Upsilon} \sup _{x \in \Omega} I(d(x)),
$$

and $\Upsilon=\left\{d \in C(\bar{\Omega}, \mathbb{B})|d|_{\partial \Omega}=i d\right\}$, where $i d$ denotes the identity operator.

Set

$$
\widetilde{\mathbb{B}}=\left\{x \in \mathbb{B} \mid x_{-k}=x_{k}, \forall k \in \mathbb{Z}\right\} .
$$

We have $\widetilde{\mathbb{B}}=Z$, then

$$
x_{k}=\sum_{i=1}^{[(s M-1) / 2]} q_{i} \sin \frac{\omega i}{s} k, \quad \forall k \in \mathbb{Z} .
$$

Lemma 2. Suppose that $G(t, x)$ satisfies $\left(G_{1}\right)-\left(G_{5}\right)$. Then,

$$
I(x)=\frac{1}{2} \sum_{k=1}^{s M}\left(\Delta^{n} x_{k-1}\right)^{2}-\sum_{k=1}^{s M} G\left(k, x_{k}\right)
$$

is bounded from above in $\mathbb{B}$.

Proof. For any $x \in \mathbb{B}$, by $\left(G_{2}\right)$, we have

$$
\begin{aligned}
I(x) & =\frac{1}{2} \sum_{k=1}^{s M}\left(\Delta^{n} x_{k-1}, \Delta^{n} x_{k-1}\right)-\sum_{k=1}^{s M} G\left(k, x_{k}\right) \\
& =\frac{1}{2} \sum_{k=1}^{s M}\left(\Delta^{n} x_{k}, \Delta^{n} x_{k}\right)-\sum_{k=1}^{s M} G\left(k, x_{k}\right) \\
& =\frac{1}{2} y^{*} L y-\sum_{k=1}^{s M} G\left(k, x_{k}\right) \\
& \leq \frac{1}{2} \times 4\|y\|^{2}-\sum_{k=1}^{s M}\left(\varrho x^{2}-\tau\right) \\
& =2\|y\|^{2}-\varrho\|x\|^{2}+s M \tau,
\end{aligned}
$$

where $y=\left(\Delta^{n-1} x_{1}, \Delta^{n-1} x_{2}, \ldots, \Delta^{n-1} x_{s M}\right)^{*}$. Since

$$
\|y\|^{2}=\sum_{k=1}^{s M}\left(\Delta^{n-2} x_{k+1}-\Delta^{n-2} x_{k}\right)^{2} \leq 4 \sum_{k=1}^{s M}\left(\Delta^{n-2} x_{k}\right)^{2} \leq \cdots \leq 4^{n-1}\|x\|^{2},
$$

then

$$
I(x) \leq \frac{1}{2}\left(4^{n}-2 \varrho\right)\|x\|^{2}+s M \tau \leq s M \tau .
$$

The proof is finished.

Lemma 3. Suppose that $G(t, x)$ satisfies $\left(G_{1}\right)-\left(G_{5}\right)$. Then,

$$
I(x)=\frac{1}{2} \sum_{k=1}^{s M}\left(\Delta^{n} x_{k-1}\right)^{2}-\sum_{k=1}^{s M} G\left(k, x_{k}\right),
$$

satisfies the Palais-Smale condition.

Proof. Assume that $\left\{I\left(x^{(j)}\right)\right\}$ is a bounded sequence from the lower bound. Then, there is a positive constant $c_{1}>0$ such that

$$
-c_{1} \leq I\left(x^{(j)}\right), \quad \forall j \in \mathbb{N} .
$$

The proof of Lemma 2 implies that

$-c_{1} \leq I\left(x^{(j)}\right) \leq\left(4^{n}-2 \varrho\right)\left\|x^{(j)}\right\|^{2}+s M \tau, \quad \forall j \in \mathbb{N}$.

Therefore,

$$
\left\|x^{(j)}\right\|^{2} \leq \frac{c_{1}+s M \tau}{2 \varrho-4^{n}}
$$

It comes from $\varrho>\left(4^{n} / 2\right)$ that we can find a positive constant $c_{2}$ such that for any $j \in \mathbb{N},\left\|x^{(j)}\right\| \leq c_{2}$. As a consequence of this, we know that the sequence $\left\{x^{(j)}\right\}_{j \in \mathbb{N}}$ is a bounded in the finite-dimensional space $\mathbb{B}$. Thus, it has a convergent subsequence. The Palais-Smale condition is verified.

Lemma 4. Suppose that $x$ is a critical point of $I(x)$ on $\widetilde{\mathbb{B}}$. Then, $x$ is a critical point of $I(x)$ on $\mathbb{B}$.

The proof of Lemma 4 is similar to the proof of Lemma 2.2 in [12]. For simplicity, we omit its proof.

Let

$$
\Theta_{\varpi}=-\frac{s}{2\left[\left(4 \sin ^{2}(\omega \varpi / 2 s)\right)^{n}-C\right]} \sum_{k=1}^{s M} g^{2}(k, 0) .
$$

Lemma 5. Suppose that $G(t, x)$ satisfies $\left(G_{1}\right)-\left(G_{5}\right)$ and $I(x)<\Theta_{m_{s}}$. If $x$ is a critical point of $I(x)$ on $\widetilde{\mathbb{B}}$, then $x$ has a minimal period $s M$.

Proof. Suppose, for the sake of contradiction, that $x$ exists a minimal period $(s M / \omega)$. In view of the condition $\left(G_{4}\right)$, we have $\omega \geq m_{s}$.

Similarly, $x_{i}$ can be written in the form of

$$
x_{k}=\sum_{i=1}^{[(s M-\Phi) / 2 \phi]} q_{i} \sin \frac{\omega \omega i}{s} k, \quad \forall x \in \widetilde{\mathbb{B}} .
$$




$$
I(x)=\frac{1}{2} y^{*} L y-\sum_{k=1}^{s M} G\left(k, x_{k}\right) \geq 2 \sin ^{2} \frac{\omega \varpi}{2 s}\|y\|^{2}-\sum_{k=1}^{s M} G\left(k, x_{k}\right)
$$

where $y=\left(\Delta x_{1}, \Delta x_{2}, \ldots, \Delta x_{s M}\right)^{*}$. It is obvious that

$$
\|y\|^{2}=\sum_{k=1}^{s M}\left(\Delta^{n-2} x_{k+1}-\Delta^{n-2} x_{k}\right)^{2} \geq 4 \sin ^{2} \frac{\omega \omega}{2 s} \sum_{k=1}^{s M}\left(\Delta^{n-2} x_{k}\right)^{2} \geq \cdots \geq\left(4 \sin ^{2} \frac{\omega \varpi}{2 s}\right)^{n-1}\|x\|^{2} .
$$

Therefore, by $\left(G_{5}\right)$,

$$
\begin{aligned}
I(x) & \geq \frac{1}{2}\left(4 \sin ^{2} \frac{\omega \Phi}{2 s}\right)^{n}\|x\|^{2}-\left(\sum_{k=1}^{s M} g^{2}(k, 0)\right)^{1 / 2}\|x\|-\frac{C}{2}\|x\|^{2} \\
& \geq-\frac{1}{2\left[\left(4 \sin ^{2}(\omega \varpi / 2 s)\right)^{n}-C\right]} \sum_{k=1}^{s M} g^{2}(k, 0) \\
& \geq-\frac{s}{2\left[\left(4 \sin ^{2}(\omega \varpi / 2 s)\right)^{n}-C\right]} \sum_{k=1}^{s M} g^{2}(k, 0) \\
& =\Theta_{\varpi} \geq \Theta_{m_{s}} .
\end{aligned}
$$

That is, $I(x) \geq \Theta_{m_{s}}$ which is a contradiction with the condition $I(x)<\Theta_{m_{s}}$, and the proof of Lemma 5 is now complete.

\section{Proofs of the Main Theorems}

In this section, the proofs of Theorems 1-3 and Corollaries 1 and 2 are given by using the critical point theory.

Proof. of Theorem 1. In view of Lemma 2, $I(x)$ is bounded from above in $\mathbb{B}$.

Set

$$
\bar{m}=\sup _{x \in \mathbb{B}} I(x) .
$$

Thus, there is a sequence $\left\{x^{(i)}\right\}$ on $\mathbb{B}$ such that

$$
\bar{m}=\lim _{i \longrightarrow \infty} I\left(x^{(i)}\right) .
$$

In addition, by the proof of Lemma 2 , for any $x \in \mathbb{B}$,

$$
I(x) \leq \frac{1}{2}\left(4^{n}-2 \varrho\right)\|x\|^{2}+s M \tau \leq s M \tau
$$

Therefore, $\lim _{\|x\| \longrightarrow+\infty} I(x)=-\infty$. This means that $\left\{x^{(i)}\right\}$ is bounded. Consequently, $\left\{x^{(i)}\right\}$ has a convergent subsequence. We define it as $\left\{x^{\left(i_{k}\right)}\right\}$. Denote

$$
\bar{x}=\lim _{k \longrightarrow+\infty} x^{\left(i_{k}\right)} .
$$

On account of the continuity of $I(x)$ in $x$, there must be a point $\bar{x} \in \mathbb{B}, I(\bar{x})=\bar{m}$. Obviously, $\bar{x} \in \mathbb{B}$ is a critical point of $I(x)$.

For any $x \in B,\|x\| \leq \delta_{1}$, from the condition $\left(G_{1}\right)$,

$$
\begin{aligned}
I(x) & =\frac{1}{2} y^{*} L y-\sum_{k=1}^{s M} G\left(k, x_{k}\right) \\
& \geq \frac{1}{2} y^{*} L y-\rho \sum_{k=1}^{s M} x_{k}^{2} \\
& \geq 2 \sin ^{2} \frac{\pi}{s M}\|y\|^{2}-\rho\|x\|^{2},
\end{aligned}
$$

where $y=\left(\Delta x_{1}, \Delta x_{2}, \ldots, \Delta x_{s M}\right)^{*}$. It is easy to see that

$$
\|y\|^{2}=\sum_{k=1}^{s M}\left(\Delta^{n-2} x_{k+1}-\Delta^{n-2} x_{k}\right)^{2} \geq 4 \sin ^{2} \frac{\pi}{s M} \sum_{k=1}^{s M}\left(\Delta^{n-2} x_{k}\right)^{2} \geq \cdots \geq\left(4 \sin ^{2} \frac{\pi}{s M}\right)^{n-1}\|x\|^{2} .
$$

Therefore,

$$
I(x) \geq\left[\frac{\left(4 \sin ^{2}(\pi / s M)\right)^{n}}{2}-\rho\right]\|x\|^{2} .
$$

Take $c=\left[\left(\left(4 \sin ^{2}(\pi / s M)\right)^{n} / 2\right)-\rho\right] \delta_{1}^{2}$. Thus, for any $x \in \partial B_{\delta_{1}}(0) \cap B$,

$$
I(x) \geq c
$$

Consequently,

$$
\bar{m}=\sup _{x \in \mathbb{B}} I(x) \geq c>0 .
$$

Furthermore, there are positive constants $c>0$ and $\delta_{1}>0$ such that for any $x \in \partial B_{\delta_{1}}(0) \cap B$,

$$
I(x) \geq c .
$$

For any $x \in A$, note that $\sum_{k=1}^{s M}\left(\Delta^{n} x_{k-1}, \Delta^{n} x_{k-1}\right)=0$, we have 


$$
I(x)=\frac{1}{2} \sum_{k=1}^{s M}\left(\Delta^{n} x_{k-1}, \Delta^{n} x_{k-1}\right)-\sum_{k=1}^{s M} G\left(k, x_{k}\right)=-\sum_{k=1}^{s M} G\left(k, x_{k}\right) \leq 0 .
$$

Thus, $\bar{x} \notin A$ and the critical point $\bar{x}$ of $I(x)$ corresponding to the critical value $\bar{m}$ is a nontrivial periodic solution of (1) with period $s M$.

Choose $\mu \in \partial B_{1}(0) \cap B$. Denote

$$
x=t \mu+\nu, \quad \forall \nu \in B, t \in \mathbb{R} .
$$

We have

$$
\begin{aligned}
I(x) & =\frac{1}{2} \sum_{k=1}^{s M}\left(\Delta^{n} x_{k-1}, \Delta^{n} x_{k-1}\right)-\sum_{k=1}^{s M} G\left(k, x_{k}\right) \\
& \leq \frac{t^{2}}{2} \sum_{k=1}^{s M}\left(\Delta^{n} \mu_{k-1}, \Delta^{n} \mu_{k-1}\right)-\sum_{k=1}^{s M} G\left(k, t \mu_{k}+v_{k}\right) \\
& \leq \frac{t^{2}}{2} z^{*} L z-\sum_{k=1}^{s M}\left[\varrho\left(t \mu_{k}+v_{k}\right)^{2}-\tau\right] \\
& \leq 2 t^{2}\|z\|^{2}-\varrho \sum_{k=1}^{s M}\left(t \mu_{k}+v_{k}\right)^{2}+s M \tau \\
& =2 t^{2}\|z\|^{2}-\varrho t^{2}-\varrho\|\nu\|^{2}+s M \tau,
\end{aligned}
$$

where $z=\left(\Delta \mu_{1}, \Delta \mu_{2}, \ldots, \Delta \mu_{s M}\right)^{*}$. Since

$$
\|z\|^{2}=\sum_{k=1}^{s M}\left(\Delta^{n-2} \mu_{k+1}-\Delta^{n-2} \mu_{k}\right)^{2} \leq 4 \sum_{k=1}^{s M}\left(\Delta^{n-2} \mu_{k}\right)^{2} \leq \cdots \leq 4^{n-1},
$$

then

$$
I(x) \leq\left(\frac{4^{n}}{2}-\varrho\right) t^{2}-\varrho\|\nu\|^{2}+s M \tau \leq-\varrho\|\nu\|^{2}+s M \tau .
$$
that

Accordingly, there exists a positive number $\varsigma>\delta_{1}$ such

$$
I(x) \leq 0, \quad \forall x \in \partial \Omega,
$$

where $\Omega=\left(\bar{B}_{\zeta}(0) \cap \mathbb{B}_{1}\right) \oplus\{t \mu \mid 0<t<\varsigma\}$. Applying the linking theorem, $I(x)$ has a critical value $c_{0} \geq c>0$, where

$$
c_{0}=\inf _{d \in \Upsilon} \sup _{x \in \Omega} I(d(x)),
$$

and $\Upsilon=\left\{d \in C(\bar{\Omega}, \mathbb{B})|d|_{\partial \Omega}=i d\right\}$.

Similar to the Proof of Theorem 1 in [22], we can prove that (1) has at least two $s M$-periodic nontrivial solutions and so we omit it. By Lemma 5, it suffices to prove that

$$
I(x)<\Theta_{m_{s}}, \quad \forall x \in \widetilde{\mathbb{B}} .
$$

From $\left(G_{3}\right)$,
$G(k, x)=g(k, 0) x+\frac{1}{2} \times \frac{\partial^{2} G(k, \varphi x)}{\partial x^{2}} x^{2} \geq g(k, 0) x+\frac{D}{2} x^{2}, \quad \forall|x| \leq \kappa$.

Thus,

$$
\begin{aligned}
I(x) & =\frac{1}{2} \sum_{k=1}^{s M}\left(\Delta^{2} x_{k}, \Delta^{2} x_{k}\right)-\sum_{n=1}^{s M} G\left(k, x_{k}\right) \\
& \leq \frac{1}{2} \sum_{k=1}^{s M}\left(\Delta^{2} x_{k}, \Delta^{2} x_{k}\right)-\frac{D}{2} \sum_{k=1}^{s M} x_{k}^{2}-\sum_{k=1}^{s M} g(k, 0) x_{k}, \quad \forall|x| \leq \kappa .
\end{aligned}
$$

Choose

$$
x_{k}=\kappa \sin \frac{\omega k}{s} .
$$

It comes from $g(-k, 0)=g(k, 0) \quad$ and $g(k+M, 0)=g(k, 0)$ that

$$
g(k, 0)=\sum_{i=1}^{[(M-1) / 2]} p_{i} \sin \frac{2 i \pi}{M} k=\sum_{i=1}^{[(M-1) / 2]} p_{i} \sin \frac{2 i \pi}{s M} s k,
$$

where $p_{i}$ is a constant. In addition,

$$
\sum_{k=1}^{s M} g(k, 0) x_{k}=\sum_{i=1}^{[(M-1) / 2]} \kappa a_{i} \sum_{k=1}^{s M} \sin \frac{2 i \pi}{s M} s k \cdot \sin \frac{2 \pi}{s M} k=0 .
$$

Consequently,

$$
I(x) \leq 2\left[\left(4 \sin ^{2} \frac{\omega}{2 s}\right)^{n}-D\right]\|x\|^{2} .
$$

It is easy to see that

$$
\|x\|=\kappa\left(\frac{s \pi}{\omega}\right)^{1 / 2} .
$$

Thus,

$$
I(x)=\frac{2\left[\left(4 \sin ^{2}(\omega / 2 s)\right)^{n}-D\right] \kappa^{2} s \pi}{\omega}<\Theta_{m_{s}} .
$$

The Proof of Theorem 1 is completed.

Proof. of Corollary 1. Since $s$ is a prime integer and $s>0$, it is easy to see that $m_{s}=s$. Therefore,

$$
\sum_{k=1}^{s M} g^{2}(k, 0)<\frac{4 \pi \kappa^{2}\left[\left(4 \sin ^{2}(\omega / 2)\right)^{n}-C\right]\left[D-\left(4 \sin ^{2}(\omega / 2 s)\right)^{n}\right]}{\omega} \text {. }
$$

In virtue of Theorem 1, the conclusion of Corollary 1 is obtained. The proof of Corollary 1 is fulfilled. 
Proof of Theorem 2. In fact, it is evident that condition $\left(G_{6}\right)$ implies $\left(G_{1}\right)$ and condition $\left(G_{7}\right)$ implies $\left(G_{2}\right)$. As a result of Theorem 1, Theorem 2 holds. The result of Theorem 2 is achieved.

Remark 1. Similar to the proof of Theorem 1, we can also prove that Theorem 3 is right. For simplicity, we omit its proof. Thanks to Theorem 3, the conclusion of Corollary 2 is obviously accomplished.

Remark 2. A real example is given by using the main results of this paper.

For $n=2$ and $s=4$, assume that

$$
\Delta^{4} x_{k-2}=\frac{1}{6} x_{k}^{2} \sin \left(\frac{2 k \pi}{5} x_{k}\right), \quad k \in \mathbb{Z} .
$$

We have

$$
\begin{aligned}
\omega & =\frac{2 \pi}{5}, \\
m_{s} & =2, \\
M & =5, \\
g(t, x) & =\frac{1}{6} x^{2} \sin \left(\frac{2 t \pi}{5} x\right) .
\end{aligned}
$$

It is easy to verify that all the assumptions of Theorem 1 are satisfied. Consequently, (77) possesses at least two nontrivial periodic solutions with minimal period (20).

\section{Data Availability}

This paper is purely theoretical, so there are no supporting data.

\section{Conflicts of Interest}

The authors declare that there are no conflicts of interest regarding the publication of this paper.

\section{Authors' Contributions}

All authors contributed equally and significantly to writing this manuscript. All authors read and approved the final manuscript.

\section{Acknowledgments}

This project was supported by the "Innovation and Strengthen University" Project of Guangzhou Maritime University (D234, F320519, A330106, B510647, F321430, and F321108).

\section{References}

[1] P. Chen and X. H. Tang, "Periodic solutions for a differential inclusion problem involving the $p(t)$-laplacian," Advances in Nonlinear Analysis, vol. 10, no. 1, pp. 799-815, 2021.
[2] C. J. Guo, R. P. Agarwal, C. J. Wang, and D. O'Regan, “The existence of homoclinic orbits for a class of first order superquadratic hamiltonian systems," Memoirs on Differential Equations and Mathematical Physics, vol. 61, pp. 83-102, 2014.

[3] C. Guo, D. O’Regan, Y. Xu, and R. P. Agarwal, "Existence of homoclinic orbits for a class of first-order differential difference equations," Acta Mathematica Scientia, vol. 35, no. 5, pp. 1077-1094, 2015.

[4] T. He, P. Guo, and L. Liu, "Multiple constant sign and nodal solutions for nonlinear nonhomogeneous elliptic equations depending on a parameter," Calculus of Variations and Partial Differential Equations, vol. 60, no. 2, p. 82, 2021.

[5] X. Tang and S. Chen, "Ground state solutions of NehariPohozaev type for Schrödinger-Poisson problems with general potentials," Discrete \& Continuous Dynamical Systems-A, vol. 37, no. 9, pp. 4973-5002, 2017.

[6] X. Tang, S. Chen, X. Lin, and J. Yu, "Ground state solutions of Nehari-Pankov type for Schrödinger equations with local super-quadratic conditions," Journal of Differential Equations, vol. 268 , no. 8 , pp. 4663-4690, 2020.

[7] H. Bin, "Periodic solutions of second-order difference problem with potential indefinite in sign," Abstract and Applied Analysis, vol. 2013, Article ID 517036, 8 pages, 2013.

[8] X. Liu, Y. B. Zhang, and H. P. Shi, "Periodic and subharmonic solutions for a $2 n$ th-order nonlinear difference equation," Hacettepe Journal of Mathematics and Statistics, vol. 44, no. 2, pp. 357-368, 2015.

[9] J. Leng, "Periodic and subharmonic solutions for $2 n$ th-order $\phi c$-laplacian difference equations containing both advance and retardation," Indagationes Mathematicae, vol. 27, no. 4, pp. 902-913, 2016.

[10] X. He, "Infinitely many homoclinic orbits for $2 n$ th-order nonlinear functional difference equations involving the p-laplacian," Abstract and Applied Analysis, vol. 2012, no. 2, 20 pages, Article ID 297618, 2012.

[11] G. H. Lin and Z. Zhou, "Periodic and subharmonic solutions for a $2 n$ th-order difference equation containing both advance and retardation with $\phi$-laplacian," Advances in Difference Equations, vol. 2014, no. 74, pp. 1-14, 2014.

[12] F. Xia, "Existence of periodic solutions for higher order difference equations containing both many advances and retardations," Revista de la Real Academia de Ciencias Exactas, Físicas y Naturales. Serie A. Matemáticas, vol. 112, no. 1, pp. 239-249, 2018.

[13] P. H. Rabinowitz, "Periodic solutions of Hamiltonian systems," Communications on Pure and Applied Mathematics, vol. 31, no. 2, pp. 157-184, 1978.

[14] A. Ambrosetti and G. Mancini, "Solutions of minimal period for a class of convex Hamiltonian systems," Mathematische Annalen, vol. 255, no. 3, pp. 405-421, 1981.

[15] V. Benci and D. Fortunato, "Estimate of the number of periodic solutions via the twist number," Journal of Differential Equations, vol. 133, no. 1, pp. 117-135, 1997.

[16] I. Ekeland and H. Hofer, "Periodic solutions with prescribed minimal period for convex autonomous hamiltonian systems," Inventiones Mathematicae, vol. 81, no. 1, pp. 155-188, 1985.

[17] H. Bin and Z. Huang, "Estimate of number of periodic solutions of second-order asymptotically linear difference system," Abstract and Applied Analysis, vol. 2013, Article ID 707686, 8 pages, 2013.

[18] T. He, W. Yang, H. Zhao, and Y. F. Lei, "Positive periodic solutions for higher order functional difference systems 
depending on a parameter," Mediterranean Journal of Mathematics, vol. 10, no. 2, pp. 791-806, 2013.

[19] T.-S. He, M. Zhang, K.-H. Liang, and P.-F. Guo, "Standing waves for discrete nonlinear Schrödinger equations with nonperiodic bounded potentials," Acta Mathematicae Applicatae Sinica, English Series, vol. 35, no. 2, pp. 374-385, 2019.

[20] J. H. Leng, "Existence of periodic solutions for a higher order nonlinear difference equation," Electronic Journal Differential Equations, vol. 2016, no. 134, pp. 1-10, 2016.

[21] G. H. Lin, J. S. Yu, and Z. Zhou, "Homoclinic solutions of discrete nonlinear Schrödinger equations with partially sublinear nonlinearities," Electronic Journal of Differential Equations, vol. 2019, no. 96, pp. 1-14, 2019.

[22] G. Lin and Z. Zhou, "Homoclinic solutions of discrete $\phi$-laplacian equations with mixed nonlinearities," Communications on Pure and Applied Analysis, vol. 17, no. 5, pp. 1723-1747, 2018.

[23] Y. H. Long, "Multiplicity results for periodic solutions with prescribed minimal periods to discrete Hamiltonian systems," Journal of Difference Equations and Applications, vol. 17, no. 10, pp. 1499-1518, 2011.

[24] P. Mei and Z. Zhou, "Periodic and subharmonic solutions for a $2 n$ th-order $p$-laplacian difference equation containing both advances and retardations," Open Mathematics, vol. 16, no. 1, pp. 1435-1444, 2018.

[25] P. Mei, Z. Zhou, and G. Lin, "Periodic and subharmonic solutions for a $2 n$ th-order $\phi_{c}$-laplacian difference equation containing both advances and retardations," Discrete \& Continuous Dynamical Systems-S, vol. 12, no. 7, pp. 20852095, 2019.

[26] F. Xia, "Homoclinic solutions for second-order nonlinear difference equations with Jacobi operators," Electronic Journal of. Differential Equations, vol. 2017, no. 94, pp. 1-11, 2017.

[27] J. Yu, Y. Long, and Z. Guo, "Subharmonic solutions with prescribed minimal period of a discrete forced pendulum equation," Journal of Dynamics and Differential Equations, vol. 16, no. 2, pp. 575-586, 2004.

[28] Z. Zhou and D. Ma, "Multiplicity results of breathers for the discrete nonlinear Schrödinger equations with unbounded potentials," Science China Mathematics, vol. 58, no. 4, pp. 781-790, 2015.

[29] Z. Zhou, J. Yu, and Y. Chen, "Homoclinic solutions in periodic difference equations with saturable nonlinearity," Science China Mathematics, vol. 54, no. 1, pp. 83-93, 2011.

[30] P. H. Rabinowitz, Minimax Methods in Critical Point Theory with Applications to Differential Equations, American Mathematical Society, Providence, RI, USA, 1986.

[31] P. Agarwal, S. K. Q. Al-Omari, and P. Park, "An extension of some variant of Meijer type integrals in the class of Boehmians," Journal of Inequalities and Applications, vol. 70, no. 1, pp. 1-10, 2016. 